\title{
In Vivo Assessment of Pulmonary Arterial Wall Fibrosis by Intravascular Optical Coherence Tomography in Pulmonary Arterial Hypertension: A New Prognostic Marker of Adverse Clinical Follow-Up ${ }^{\S}$
}

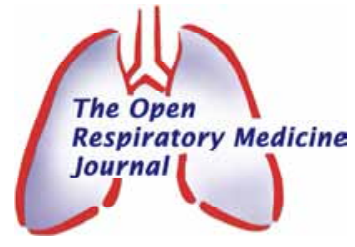

\author{
Enric Domingo ${ }^{*}, 1,2$, Juan C. Grignola ${ }^{3}$, Rio Aguilar ${ }^{2}$, María Angeles Montero ${ }^{4}$, \\ Christian Arredondo ${ }^{1}$, Manuel Vázquez ${ }^{1}$, Manuel López-Messeguer ${ }^{5}$, \\ Carlos Bravo ${ }^{5}$, Nadia Bouteldja ${ }^{1}$, Cristina Hidalgo ${ }^{1}$ and Antonio Roman ${ }^{5}$
}

\author{
${ }^{1}$ Area del Cor, Hospital Universitari Vall d'Hebron, Spain \\ ${ }^{2}$ Dept Fisiología Universitat Autonoma Barcelona, Spain \\ ${ }^{3}$ Dept de Fisiopatología, Hospital de Clínicas, Universidad de la República, Montevideo, Uruguay \\ ${ }^{4}$ Servei de Anatomia Patológica, Hospital Universitari Vall d'Hebron, Spain \\ ${ }^{5}$ Servei de Pneumologia i CIBERES, Hospital Universitari Vall d'Hebron, Spain
}

\begin{abstract}
Background: The aim is to correlate pulmonary arterial (PA) remodeling estimated by PA fibrosis in PA hypertension (PAH) with clinical follow-up. Histology of PA specimens is also performed.

Methods: 19 patients, aged 54 \pm 16 (4 men), functional class II-III were studied with right heart catheterization, PA Intravascular Ultrasound and optical coherence tomography (OCT) in inferior lobe segment. PA wall fibrosis was obtained by OCT ( area of fibrosis/PA cross sectional area $\times 100$ ). Patients follow-up was blind to OCT. Events were defined as mortality, lung transplantation, need of intravenous prostaglandins or onset of right ventricular failure.

Results: OCT measurements showed high intra- and interobserver agreement. There was a good correlation between OCT and histology in PA fibrosis from explanted lungs. Area of fibrosis was $1.4 \pm 0.8 \mathrm{~mm}^{2}, \%$ fibrosis was $22.3 \pm 8$. Follow-up was 3.5 years (2.5-4.5). OCT \%Fib was significantly correlated with PA capacitance $(\mathrm{r}=-0.536)$ and with pulmonary vascular rsistance $(\mathrm{r}=0.55)$. Patients were divided according to the median value of PA fibrosis. There were 10 patients with a high $(\geq 22 \%)$ and 9 with a low fibrosis $(<22 \%)$. Events occurred in 6 ( 1 death, 1 lung transplantation, 2 intravenous prostaglandins, 2 right heart failure) out of 10 patients with high and in 0 out of 9 patients with low fibrosis $(\mathrm{p}<0.01)$.

Conclusions: In PAH, the severity of PA remodeling assessed by OCT wall fibrosis was significantly predictive of severely unfavorable clinical outcome. In vivo assessment of pulmonary arterial wall fibrosis by intravascular OCT in $\mathrm{PAH}$ is a promising new prognostic marker of adverse clinical outcome.
\end{abstract}

Keywords: Catheterization, pulmonary heart disease, prognosis, remodeling.

\section{INTRODUCTION}

Pulmonary arterial hypertension (PAH) is a rare and severe condition characterized by vascular proliferation and remodeling of the small pulmonary arterial wall. The histological findings in PAH include intimal hyperplasia, medial hypertrophy and adventitial proliferation/fibrosis. Currently the risk-to-benefit ratio of open lung tissue biopsy in $\mathrm{PAH}$ is unfavorable. However, intimal thickening by

\footnotetext{
*Address correspondence to this author at the Cardiology Department, Hospital Universitari Vall d'Hebron, Pg Vall d'Hebron 119-129, 08035, Barcelona, Spain; Tel: (+34) 93 2746155; Fax: (+34) 93 2746001;

E-mail: edrcg@hotmail.com
}

${ }^{\S}$ This study was presented in part in the Scientific Session of the American Heart Association (AHA), Nov 12-16th. Orlando, FL; USA. A9223. Circulation 124 (21, Suppl): A9223; 2011. fibrosis appears to predict shorter survival from the time of biopsy $[1,2]$.

Palevsky et al. showed that an intimal area of more than $18 \%$ of the vascular cross-sectional area had an $85 \%$ predictive value for identifying poor outcome [3].

Therefore, accurate detection and quantification of intimal fibrosis would be important in the assessment of PA remodeling and disease prognosis.

In addition, the histological remodelling process of the arterial wall has been characterized by narrowing and stiffening. Pulmonary arterial stiffening, which can occur both proximal and distal PAs, has gained increasing recognition due to its significant contribution to RV afterload and clinical relevance in PAH [4-6]. 
Remarkable advances in the different vascular imaging techniques such as computed magnetic resonance, intravascular ultrasound (IVUS) or optical coherence tomography (OCT) provide an opportunity to gain insight into the histopathology that could previously only be obtained through open lung biopsy.

Intravascular Ultrasound (IVUS) has been validated as a reliable method for describing pulmonary vessel wall morphology $[4,7,8]$.

Optical Coherence Tomography (OCT) is a new highresolution imaging modality that has proven its superiority over IVUS for characterization of coronary atherosclerotic plaque $[9,10]$.

OCT utilizes a near infrared light source $(1300 \mathrm{~nm}$ of wavelength) in combination with advanced fiber-optics to create a dataset of the artery wall. The use of infrared light allows for a ten-fold higher image resolution but at the expense of a reduced penetration depth (2-3 vs 4-8 mm) [11]. According to our knowledge, there is scarce and poorly analyzed literature on the use of OCT in PAH $[12,13]$.

The objective of this study is to investigate the ability and accuracy of OCT to detect and quantify intimal fibrosis, as a marker of histological remodeling of the pulmonary arterial wall in patients with $\mathrm{PAH}$, and to analyze its relation to right ventricular afterload and clinical prognosis.

\section{METHODS}

\section{Study Population}

Nineteen consecutive patients with stable (no change in clinical status or medication in the last 6 months) PAH attending the Pulmonary Hypertension Unit outpatient clinic of our hospital were recruited for undergoing cardiac catheterization at our institution (January 2007 - September 2008). The only exclusion criteria were refusal to participate in the study or having PAH in functional class IV. The study had been approved by the institutional ethics committee, and a written informed consent had been obtained from every patient. Basal clinical and hemodymanic characteristics are shown in Table $\mathbf{1}$.

The patients were aged 25-81 years, in functional class 23 , with no clinical and pharmacological changes in the last 46 months. The diagnosis of PAH was made according to the standard algorithm including a right heart catheterization. All patients were treated according to current guidelines.( 3 on bosentan, 10 on sildfenafil, 4 on bosentan and sildenafil, 1 on bosentan, sildenafil and epoprostenol, and 1 on bosentan, sildenafil and iloprost ).

\section{Hemodynamic and IVUS Assessments}

Right heart catheterization is part of the routine followup of these patients at our institution. IVUS and OCT were performed for the purposes of the study. All patients underwent right heart catheterization in supine position and breathing room air. A 7 F Swan-Ganz catheter (Edwards Lifesciences, USA) was inserted into a brachial vein and a 5 $\mathrm{F}$ end-hole catheter was inserted into the right radial artery. Catheters were connected to fluid-filled transducers and zeroed at the atmospheric pressure. Right atrial pressure, pulmonary arterial pressure (PAP), pulmonary arterial occlusion pressure (PAOP) and systemic arterial pressure were measured. Cardiac output and cardiac index were obtained from the Fick method and assumed basal oxygen consumption. Pulmonary vascular resistance index (PVRI) was calculated as mean arterial pulmonary pressure minus PAOP/cardiac output $\mathrm{x}$ body surface area. Pulmonary vascular capacitance was estimated as stroke volume/pulse pressure [5,14]

Table 1. Hemodynamic, IVUS and OCT Data

\begin{tabular}{|c|c|}
\hline Female, n (\%) & $15(79)$ \\
\hline Age (years) & $54 \pm 16$ \\
\hline \multicolumn{2}{|l|}{ WHO, n (\%) } \\
\hline Class II & $9(47)$ \\
\hline Class III & $10(53)$ \\
\hline \multicolumn{2}{|l|}{ Diagnoses, n (\%) } \\
\hline IPAH & $14(74)$ \\
\hline PAH associated with collagen vascular disease & $3(16)$ \\
\hline PAH associated with HIV & $2(10)$ \\
\hline 6MWT (m) & $384 \pm 99$ \\
\hline Mean PAP (mmHg) & $46 \pm 14$ \\
\hline Pulse PAP (mmHg) & $44 \pm 15$ \\
\hline PVRI (wood units.m²) & $16.7 \pm 7.4$ \\
\hline $\mathrm{Cp}(\mathrm{ml} / \mathrm{mmHg})$ & $1.33 \pm 0.8$ \\
\hline CI (liters $/ \mathrm{min} / \mathrm{m}^{2}$ ) & $2.2 \pm 0.5$ \\
\hline $\mathrm{SVI}\left(\mathrm{ml} / \mathrm{m}^{2}\right)$ & $30 \pm 9$ \\
\hline RAP (mmHg) & $8 \pm 3$ \\
\hline PAOP (mmHg) & $9 \pm 2$ \\
\hline Mixed VOS (\%) & $64 \pm 7$ \\
\hline IVUSp (\%) & $23.6 \pm 11$ \\
\hline Elastic Modulus (mmHg) & $200 \pm 85$ \\
\hline$\%$ Fibrosis (OCT) & $22.3 \pm 8$ \\
\hline Fibrosis Area $\left(\mathrm{mm}^{2}\right)$ & $1.4 \pm 0.8$ \\
\hline
\end{tabular}

Data are mean \pm SD. CI: cardiac index; Cp: capacitance index; IVUSp: IVUS pulsatility; Mixed VOS: mixed venous oxygen saturation; PAOP: pressure arterial oclusion pressure; PAP: pulmonary arterial pressure; RAP: right atrial pressure.

IVUS was done with an Eagle Eye Gold catheter $20 \mathrm{MHz}$, 3.5F (Volcano Corporation, USA). Images were obtained from a segmental PA of the inferior lobe (elastic PA between 2-3 mm) [15]. Diastolic and systolic cross-sectional areas of the studied segment were analyzed off-line by two observers unaware of clinical and hemodynamic findings. We estimated IVUS pulsatility as (systolic - diastolic lumen area/diastolic lumen area $\times 100)$ and elastic modulus as (pulse pressure/pulsatility) $[4,15,16]$. Intra- and interobserver validation of IVUS measurements in our laboratory had already been published [6].

\section{Time Domain OCT Acquisition and Analysis}

After hemodynamic and IVUS measurements, the OCT acquisition was performed using a commercially available 
system for intracoronary imaging (LightLab Imaging, Westford MA, USA) [11].

An Helios coaxial occlusion balloon catheter and a LightLab ImageWire (LightLab Imaging Inc, Westford MA, USA) was advanced over a 0,014" guidewire into the same pulmonary artery branch where the IVUS images were obtained, using an exchange guidewire system and X-ray control. This method guarantees that IVUS, OCT and PA pressure are obtained at the same level of the studied PA branch. Images were acquired during a pullback rate of 1.0 $\mathrm{mm} / \mathrm{s}$. Occlusion and saline flush technique was used. PA flow is stopped by inflating the proximal occlusion balloon and flushing a crystalloid solution through the end-hole of the balloon catheter in order to run off blood and obtain high quality images. The size of the studied arteries was within the published penetration depth range of the OCT [17].

According to the study from Yabushita et al. [18], fibrosis is represented by OCT as intimal thickening with a high reflectivity, low attenuation and homogenous signal. In the same image the inner lumen area and the area limited by the outer boundary of fibrosis are measured. Subtraction of these two areas represents fibrosis area. Percentage of fibrosis is calculated as the ratio between the fibrosis area / lumen cross-sectional area (\% fibrosis).

Reproducibility of the OCT fibrosis area was assessed in a random sample of $10 \mathrm{PAH}$ patients of the study population.. Bland-Altman analysis was performed to evaluate the intraobserver and interobserver agreement. The OCT measurements were repeated blindly a few days later by the same observer and by a second independent observer, on the same cross-sectional image of the OCT pullback. The results are expressed as intraclass correlation coefficient.

\section{Pathology Study}

One pulmonary artery from a patient with PAH and one from a normal lung were studied with OCT and histology. The arteries were dissected from two explanted lungs, one from a patient with $\mathrm{PAH}$ receiving pulmonary transplantation and the other from a patient with healthy lungs who died from another cause. Two $4 \mathrm{~cm}$-long arterial segments were sent for OCT imaging. After the OCT study, the arteries were sectioned every $2 \mathrm{~mm}$, embedded in paraffin, sectioned with the microtome at $5 \mu \mathrm{m}$ and stained with Hematoxylin-Eosin and Masson Trichrome for wall fibrosis assessment. OCT imaging was performed with the same OCT imaging catheter with the pulmonary artery submerged in saline. Automatic pull-back at a constant speed was performed along the length of the artery and used to calculate the precise location of the studied PA segment. OCT and histological images were thus obtained from the same PA section.

\section{Clinical Follow-Up}

Patients were prospectively followed up every six months. No patients were lost to follow-up. Physicians who carried out the follow-up were blind to the IVUS and OCT results. Prior to analysis, patients had been divided into 2 groups, low and high degree of fibrosis, according to the median value of PA wall OCT fibrosis percentage.
The outcome was clinical deterioration, that had been initially defined in terms of either mortality, lung transplantation, need of intravenous prostaglandins or onset of clinical right ventricular failure.

\section{Statistical Analysis}

Both measured and calculated data were expressed as mean \pm standard deviation. Data analysis was carried out with SPSS 17.0 for Windows. All subjects were included in the analysis. Results were considered significant if $\mathrm{p} \leq 0.05$. Student's t-test for independent samples was used to evaluate differences between groups; chi-squared was used for analyzing categorical variables (proportions), and Pearson's test for correlations. Bland-Altman analysis was used to evaluate inter- and intraobserver agreement. The KaplanMeier method was used to obtain survival curves.

\section{RESULTS}

\section{Hemodynamics, OCT and IVUS in PAH Patients}

Table 1 shows clinical data and mean values for hemodynamic, IVUS and OCT variables of all patients. A typical OCT image of a PA in a patient with pulmonary arterial hypertension is shown in Fig. (1). Measurements of both maximal and minimal areas showed a high intra and interobserver agreement. Intraclass intraobserver correlation coefficients were: 0.997 (CI95\%: 0.989-0.999) and 0.999 (CI95\%:0.996-0.999) and the interclass interobserver correlation coefficients were: 0.998 (CI95\%: 0.993-0.999) and 0.999 (CI95\%:0.998-0.999) for maximal and minimal areas, respectively.

OCT percentage of fibrosis was better correlated with capacitance $(\mathrm{r}=-0.53 ; \mathrm{p}=0.02)$ than with PVRI $(\mathrm{r}=0.55$; $\mathrm{p}=0.01$ ) (Fig. 2). Neither IVUS pulsatility nor elastic modulus were correlated with OCT. After dividing the cohort of patients according to the median of elastic modulus (203 $\mathrm{mmHg}$ ), no differences in percentage of fibrosis were found between high and low wall stiffness groups $(21 \pm 5 \mathrm{vs}$ $21 \pm 9 \%$, NS). After dividing the cohort of patients according to the median of OCT fibrosis $(22 \%)$, mean PAP and PVRI were higher $(\mathrm{p}<0.05)$ and $\mathrm{Cp}$ was lower $(\mathrm{p}=$ 0.07 ) in the patients with higher OCT fibrosis. However, no differences in IVUSp and EM were found between high and low PA fibrosis groups $(25 \pm 14$ vs $27 \pm 17 \%$; and $197 \pm$ 100 vs $194 \pm 68 \mathrm{mmHg}$; respectively).

\section{OCT and Histological Fibrosis}

Fig. (3) shows the excellent agreement between the assessment of PA wall fibrosis by OCT and histology in a patient without $\mathrm{PH}$ (no fibrosis) and in a patient with $\mathrm{PAH}$ (severe fibrosis).

\section{Clinical Follow-Up}

All patients were prospectively followed up for 3.5 years (2.5-4.5 years). Patients were divided into 2 groups, low $(16 \pm 4)$ and high $(29 \pm 6 \%)$ degree of fibrosis, according to the median value of PA wall OCT fibrosis percentage $(22 \%)$. Mean follow-up time was not significantly different between patients with severe or non-severe fibrosis. There were 10 patients with a high degree of fibrosis $(\geq 22 \%)$ and 9 with a 
(a)

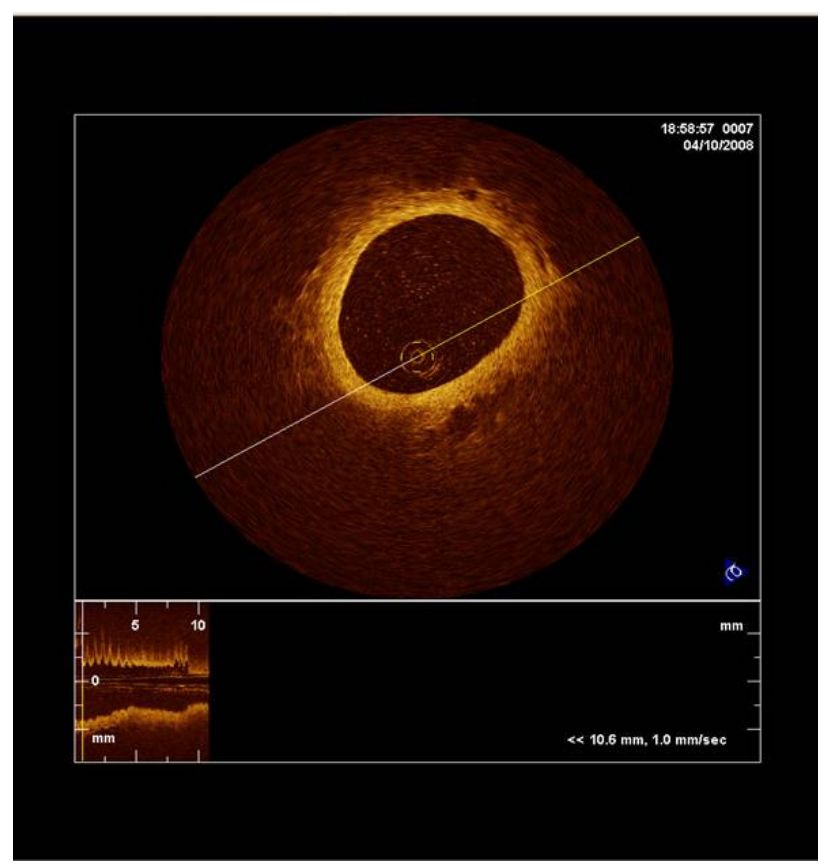

(b)

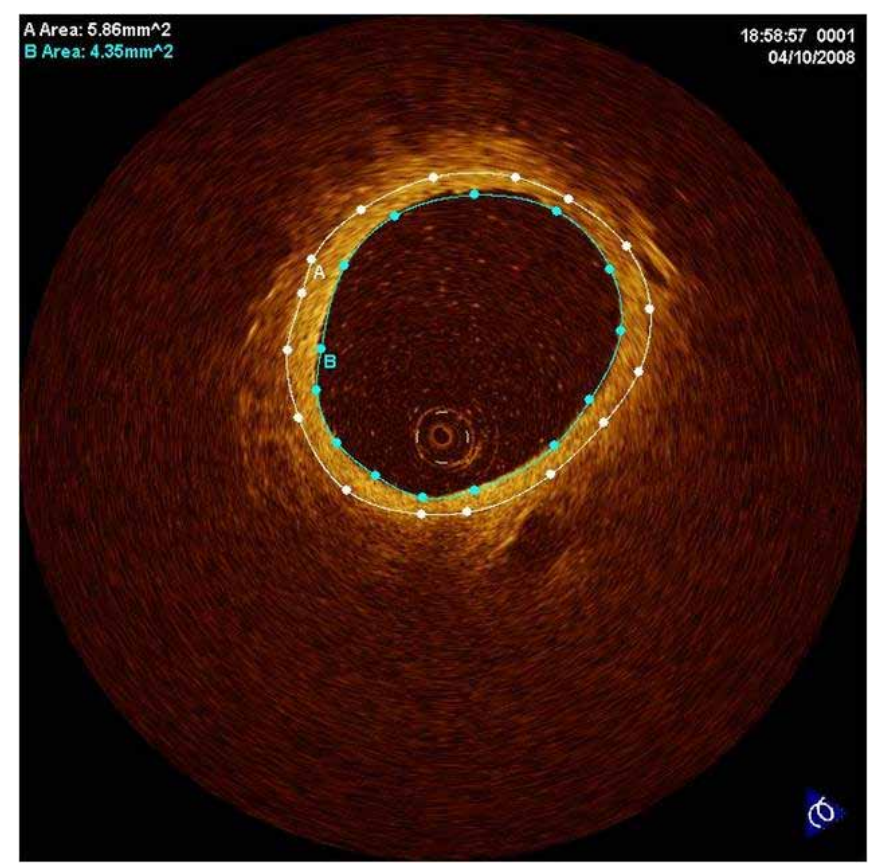

Fig. (1). In vivo OCT image of a pulmonary artery in a patient with Pulmonary Arterial Hypertension (a). Calculation of the amount of fibrosis of the arterial wall (b).
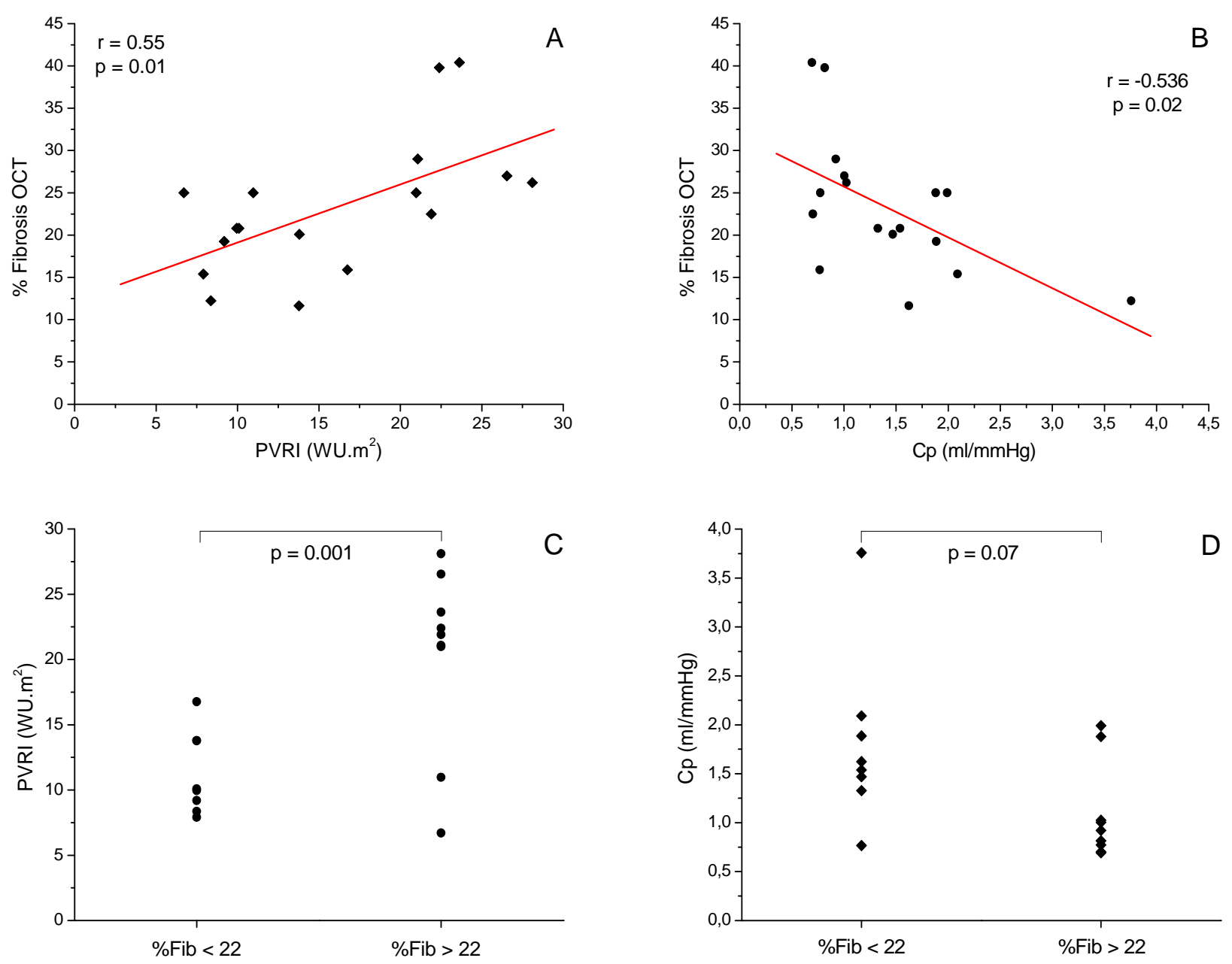

Fig. (2). Pearson correlations between pulmonary vascular resistance index (PVRI) (a) and PA capacitance (b) and \% of fibrosis. Dot diagram of PVRI (c) and Cp (d) in all patients studied grouped according to \% of fibrosis. 
(a)

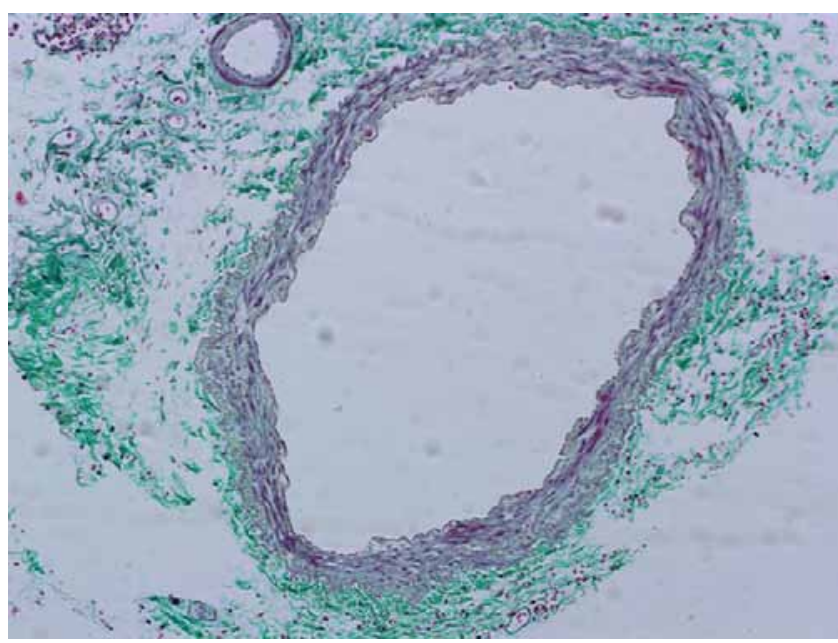

(c)

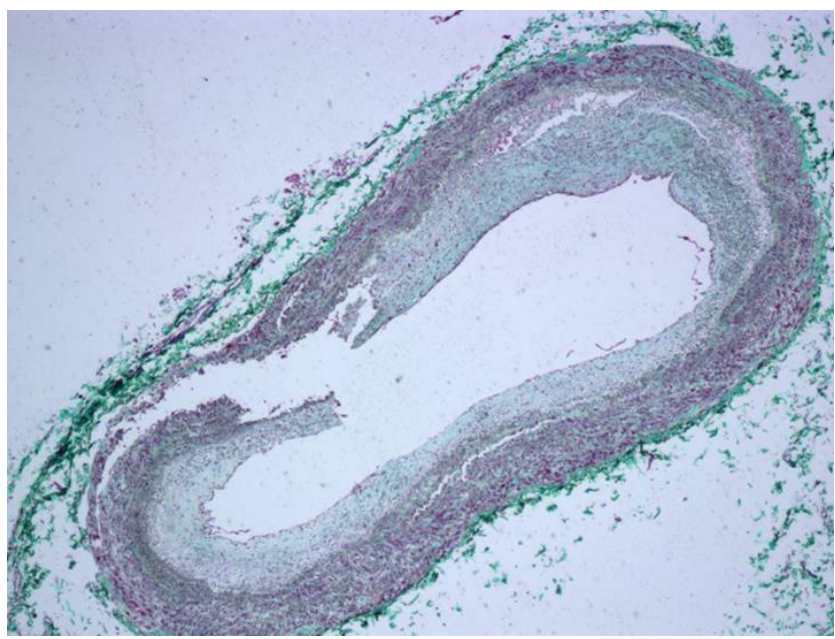

(b)

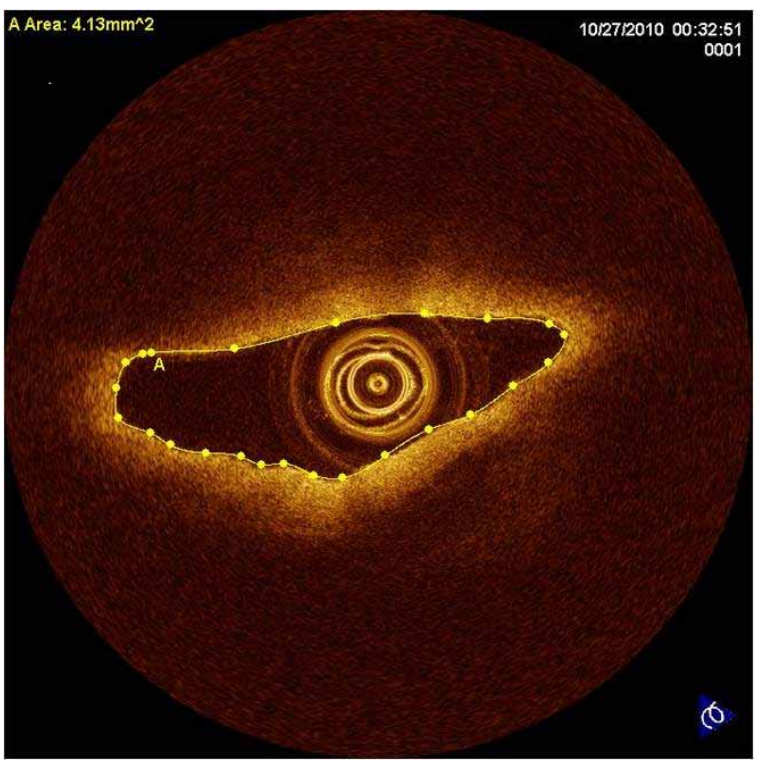

(d)

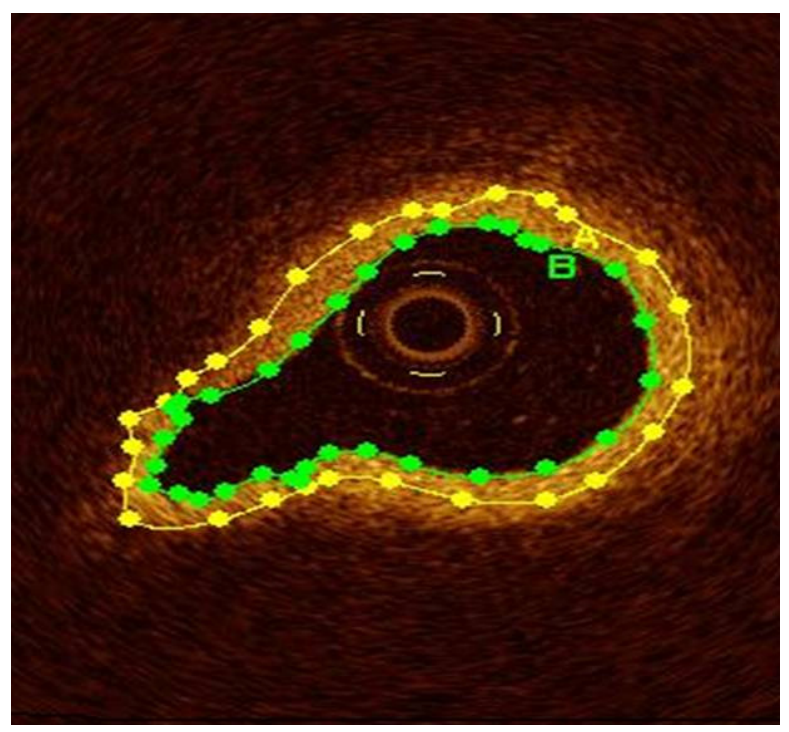

Fig. (3). Cross-section of a medium-sized pulmonary artery from a patient without pulmonary hypertension showing a medium-sized pulmonary artery without hypertensive changes (Masson Trichrome, $x$ 100) (a). OCT image of the same cross-section where no fibrosis is appreciated (b). Cross-section of a medium-sized pulmonary artery from a patient with pulmonary hypertension showing severe intimal fibrosis (Masson Tricrome, x 100) (c). OCT image of the same cross-section where the area of fibrosis has been marked ( area between green and yellow lines ) (d).

lower degree of fibrosis $(<22 \%)$. Clinical deterioration, as previously defined, were present in 6 patients $(1$ death, 1 lung transplantation, 2 intravenous prostaglandins, 2 right heart failure) out of 10 patients with severe fibrosis and in 0 out of 9 patients with less fibrosis $(\mathrm{p}<0.01)$. In Kaplan-Meier analysis, mean time to event was markedly different between both groups of patients (Log-Rank test p=0.011) (Fig. 4).

\section{DISCUSSION}

This is the first study to assess the ability of OCT for detecting and estimating PA fibrosis and to correlate it with RV afterload, PA wall stiffness and clinical prognosis in patients with stable PAH. OCT provides a high-quality morphological evaluation of the elastic pulmonary vessels of patients with pulmonary hypertension. The exploration is safe, and can be undertaken at the time of right heart cardiac catheterization with a high inter and intraobserver agreement.

The excellent agreement between OCT and histological fibrosis images in the explanted PA offer additional validation for using this new technique to quantify PA wall fibrosis (Fig. 3).

Patients with a higher degree of wall intimal fibrosis showed higher steady (PVRI increase) and pulsatile (capacitance decrease) components of PA afterload. Although intimal fibrosis is associated with an increase in 
elastic modulus, the absence of correlation between structural (OCT fibrosis) and functional (IVUS elastic modulus) wall remodeling makes evident the complex interaction between the different PA wall components in the remodeling process. The increased elastic modulus not only depends on the extent of wall fibrosis. Vasoconstriction, transmural PA pressure and wall geometrical factors (thickness/radius ratio) also influence the elastic modulus. In addition, this cohort represents extremely ill patients with such a high degree of wall fibrosis that it does not allow the analysis of the relationship between fibrosis and elastic modulus throughout the whole continuum of the disease process [16,19-21].

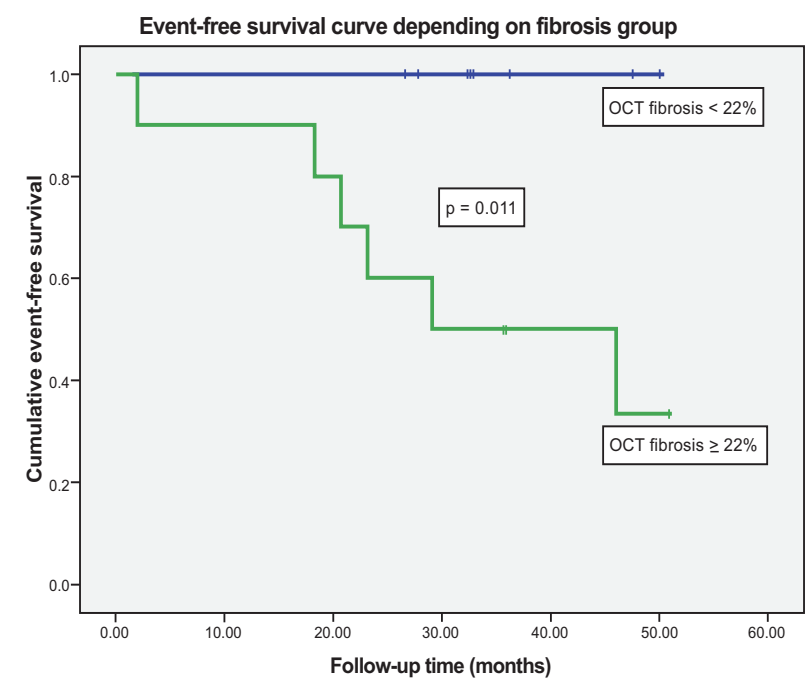

Fig. (4). Event-free Kaplan-Meier survival curve for severe fibrosis (green) and non-severe fibrosis (blue) groups of patients showing a clinically relevant and significant difference.

Palevsky et al. [3] showed that only the area of intimal fibrosis was significantly different between those patients who exhibited rapid deterioration and those $\mathrm{PH}$ who remain stable on follow-up. The mean area of intimal fibrosis was $25.6 \pm 15.8 \%$ for the group that worsened rapidly and $10 \pm$ $7.6 \%$ for stable patients, with $18 \%$ as cutoff point. This is in accordance with our data, since patients with severe fibrosis $(\geq 22 \%)$ presented more clinical events (including death and lung transplantation) than patients with less fibrosis $(<22 \%)$.

OCT only allows the assessment of capacitance vessels but not that of resistance vessels. Capacitance vessels are upstream from the resistance vessels evaluated using standard histopathologic criteria for disease severity. However the decrease in PA capacitance has been proven to be a strong predictor of mortality tn PAH [5].

Therefore the in vivo histological analysis with OCT of the severity of wall fibrosis of capacitance PA may represent a novel approach to the study of the remodeling process in these arteries. Furthermore the severity of OCT wall fibrosis observed in our patients is clearly and significantly related to clinically relevant events during mid-term follow-up, which stresses the prognostic value of the assessment of histological wall remodeling in capacitance vessels (Fig. 4).

\section{STUDY LIMITATIONS}

The pulmonary vascular illness is patchy rather than uniform throughout the lung. We estimated OCT fibrosis of PA elastic arteries of lower lobes since Bressollette et al. showed that lower lobes PA have greater wall thickness than in upper lobes [22].

A limitation of OCT is its limited penetration depth, although the vessels imaged in this study $(2-3 \mathrm{~mm})$ are in the range of OCT performance [17,23,24]. Although, there is a good correlation between histological intimal fibrosis and OCT fibrosis, additional clinical-pathological correlation studies will be important to further define PA wall tissue characteristics by OCT assessments.

This study only analyzed 19 patients. Although this may be considered a small group, PAH is an infrequent disease and the number of studied patients was large enough to obtain a significant correlation between PA wall fibrosis and mid-term clinical follow-up.

\section{CONCLUSION}

PA wall fibrosis assessed by OCT correlates with the severity of PA dynamic afterload. In PAH, the severity of PA remodeling assessed by quantification of PA wall fibrosis by OCT was significantly predictive of severely unfavorable clinical outcome. We suggest the usefulness of OCT as a novel diagnostic tool for evaluating PA structural wall remodeling in patients with PAH throughout the disease process, as a marker of prognosis and potential response to treatment [25].

\section{AUTHORS CONTRIBUTIONS}

Enric Domingo, MD, PhD was the principal investigator of the study and the corresponding author. He was responsible for the design of the study, the performance of the cardiac catheterizations, the interpretation of data and the paper writing.

Juan C. Grignola, MD, PhD was responsible for data interpretation and paper writing.

Rio Aguilar, MD was responsible for data interpretation and manuscript revision.

María Angeles Montero, MD was responsible for histological analysis.

Christian Arredondo, MD was responsible for drafting and revising the manuscript.

Manuel Vázquez was responsible for OCT and IVUS acquisition and measuring.

Manuel López-Messeguer, MD was responsible for patient selection and clinical follow-up.

Carlos Bravo, MD was responsible for patient selection and clinical follow-up.

Nadia Bouteldja, BSc was responsible for drafting and revising the manuscript.

Antonio Roman, MD was responsible for patient selection and clinical follow-up, and for drafting and revising the manuscript. 


\section{ABBREVIATIONS}

$\begin{array}{ll}\text { OCT } & =\text { Optical coherence tomography } \\ \text { IVUS } & =\text { Intravascular ultrasound } \\ \text { PA } & =\text { Pulmonary artery } \\ \text { PAH } & =\text { Pulmonary arterial hypertension } \\ \text { MWT } & =\text { Minute walking test } \\ \text { PVRI } & =\text { Pulmonary vascular resistance index } \\ \text { Cp } & =\text { Pulmonary capacitance } \\ \text { CI } & =\text { Cardiac index } \\ \text { SVI } & =\text { Stroke volume index } \\ \text { RAP } & =\text { Right atrial pressure } \\ \text { PAOP } & =\text { Pulmonary artery occlusion pressure } \\ \text { EM } & =\text { Elastic modulus }\end{array}$

\section{CONFLICT OF INTEREST}

The authors confirm that this article content has no conflict of interest.

\section{ACKNOWLEDGEMENTS}

The work was partially financially supported by a grant from Glaxo-Smith-Kline.

Juan C Grignola is supported by CSIC (Comisión Sectorial de Investigación Científica), Universidad de la República, Montevideo, Uruguay

\section{REFERENCES}

[1] Nicod P, Moser KM. Primary pulmonary hypertension: the risk and benefit of lung biopsy. Circulation 1989; 80: 1486-8.

[2] Heath D, Edwards JE. The pathology of hypertensive pulmonary vascular disease. Circulation 1958; 18: 533-47.

[3] Palevsky HI, Schloo BL, Pietra GG, et al. Primary pulmonary hypertension. Vascular structure, morphometry and responsiveness to vasodilator agents. Circulation $1989 ; 80 ; 1207-21$.

[4] Rodés Cabau J, Domingo E, Roman A, et al. Intravascular ultrasound of the elastic pulmonary arteries: a new approach for the evaluation of primary pulmonary hypertension. Heart 2003; 89: 311-5.

[5] Mahapatra S, Nishimura RA, Sorajja P, Cha S, McGoon MD. Relationship of pulmonary arterial capacitance and mortality in idiopathic pulmonary arterial hypertension. J Am Coll Cardiol 2006; 47: 799-803.

[6] Wang Z, Chesler NC. Pulmonary vascular wall stiffness: an important contributor to the increased right ventricular afterload with pulmonary hypertension. Pulm Circ 2011; 1: 212-23.

[7] Pandian NG, Weintraub A, Kreis A, Schwartz SL, Konstam MA, Salem DN. Intracardiac, intravascular, two-dimensional, highfrequency ultrasound imaging of pulmonary artery and its branches in humans and pulmonary artery. Circulation 1990; 81: 2007-12.
[8] Ishii M, Kato H, Kawano T, et al. Evaluation of pulmonary artery histopathologic findings in congenital heart disease: an in vitro study using intravascular ultrasound imaging. J Am Coll Cardiol 1995; 26: 272-6.

[9] Rieber J, Meissner O, Babaryka, et al. Diagnostic accuracy of optical coherence tomography and intravascular ultrasound for the detection and characterization of atherosclerotic plaque composition in ex-vivo coronary specimens: a comparison with histology. Coron Artery Dis 2006; 17: 425-30.

[10] Regar E, Schaar JA, Mont E, Virmani R, Serruys PW. Optical coherence tomography. Cardiovasc Radiat Med 2003; 4: 198-204.

[11] Gonzalo N, Tearney G, Serruys P, et al. Second generation optical coherence tomography in clinical practice. Rev Esp Cardiol 2010; 63: 893-903

[12] Hou J, Qi H, Zhang M, et al. Pulmonary vascular changes in pulmonary hypertension: optical coherence tomography findings. Circ Cardiovac Imaging 2010; 3: 344-5

[13] Shunsuke T, Yoshihiro F, Koichiro K, et al. Optical coherence tomography as a novel diagnostic tool for distal type chronic thromboembolic pulmonary hypertension. Circ J 2010; 74: 1742-4.

[14] Laurent S, Cockroft J, Van Bortel L, et al. Expert Consensus Document on Arterial Stiffness: methodological issues and clinical applications. Eur Heart J 2006; 27: 2588-602.

[15] Mintz GS, Nissen SE, Anderson WB, et al. American college of cardiology clinical expert consensus document on standards for acquisition, measurement and reporting of intravascular ultrasound studies (IVUS): a report of the american college of cardiology task force on clinical expert consensus documents. J Am Coll Cardiol 2001; 37: 1478-92.

[16] Santana DB, Barra JG, Grignola JC, Gines FF, Armentano RL. Pulmonary artery smooth muscle activation attenuates arterial dysfunction during acute pulmonary hypertension. J Appl Physiol 2005; 98: 605-13.

[17] Fujimoto JG. Optical coherence tomography for ultrahigh resolution in vivo imaging. Nat Biotechnol 2003; 21: 1361-7.

[18] Yabushita H, Bouma BE, Houser SL, et al. Characterization of human atherosclerosis by optical coherence tomography. Circulation 2002; 106: 1640-5.

[19] Lammers SR, Kao PH, Qi HJ, et al. Changes in the structurefunction relationship of elastin and its impact on the proximal pulmonary arterial mechanics of hypertensive calves. Am J Physiol 2008; 295: H1451-9.

[20] Ooi CY, Wang Z, Tabima DM, Eickhoff JC, Chesler NC. The role of collagen in extralobar pulmonary artery stiffening in response to hypoxia induced pulmonary hypertension. Am J Physiol 2010; 299: H1823-31.

[21] Pries AR, Secomb TW. Control of blood vessel structure: insights from theoretical models. Am J Physiol 2005; 288: H1010-5.

[22] Bressollette E, Dupuis J, Bonan R, Doucet S, Cernacek P, Tardif JC. Intravascular ultrasound assessment of pulmonary vascular disease in patients with pulmonary hypertension. Chest 2001; 120: 809-15.

[23] Takano M, Inami S, Jang IK, et al. Evaluation by optical coherence tomography of neointima coverage of sirolimus- eluting stent three months after implantation. Am J Cardiol 2007; 99:1033-10.

[24] Matsumoto D, Shite J, Shinke T, et al. Neointimal coverage of sirolimus-eluting stents at 6-month follow-up evaluated by optical coherence tomography. Eur Heart J 2007; 28: 961-7.

[25] Domingo E, Aguilar R, López-Meseguer M, Teixidó G, Vazquez $\mathrm{M}$, Roman A. New concepts in the invasive and non invasive evaluation of remodeling of the right ventricle and pulmonary vasculature in pulmonary arterial hypertension. Open Respir Med J 2009; 3: 31-37. 